\title{
Status of the James Webb Space Telescope Observatory
}

\author{
Mark Clampin \\ NASA Goddard Space Flight Center
}

\begin{abstract}
The James Webb Space Telescope (JWST) is the largest cryogenic, space telescope ever built, and will address a broad range of scientific goals from first light in the universe and re-ionization, to characterization of the atmospheres of extrasolar planets. Recently, significant progress has been made in the construction of the observatory with the completion of all 21 flight mirrors that comprise the telescope's optical chain, and the start of flight instrument deliveries to the Goddard Space Flight Center. In this paper we discuss the design of the observatory, and focus on the recent milestone achievements in each of the major observatory sub-systems.

Keywords: $\quad$ JWST, Space Telescopes, Infrared
\end{abstract}

\section{INTRODUCTION}

The James Webb Space Telescope (JWST) is a 6.5 meter aperture, cryogenic space telescope. It is designed to undertake a broad range of science programs focused on four major science themes (gardner et al. 2006): First light and re-ionization, which seeks to identify the first galaxies to form in the universe, and trace the ionization history of the universe; the Assembly of Galaxies will determine how galaxies and dark matter evolved to the present day; the Birth of Stars and Protoplanetary Systems will study how stars are formed, and focus on the early development of stars and the formation of protoplanetary systems; Planetary Systems and the Origins of Life will focus on the physical and chemical properties of our own solar system and extra-solar systems. JWST will succeed the Hubble Space Telescope (HST) a UV/Visible/Near-IR space telescope, and the Spitzer Space Telescope (SST), a mid to far-infrared telescope. Building on the legacy of these missions JWST's observatory architecture will deliver a major advance in discovery capability.

The primary design drivers for JWST are a large collecting area, combined with diffraction-limited imaging in the near-IR $(\geq 0.8$ Strehl @ $2 \mu \mathrm{m})$, cryogenic operating temperatures $(\sim 40 \mathrm{~K})$ to facilitate zodiacal light-limited, near and mid-infrared imaging $(1 \mu \mathrm{m}-10 \mu \mathrm{m})$, and an orbit that provides a benign thermal environment combined with continuous science operations. The telescope's 6.5 meter aperture

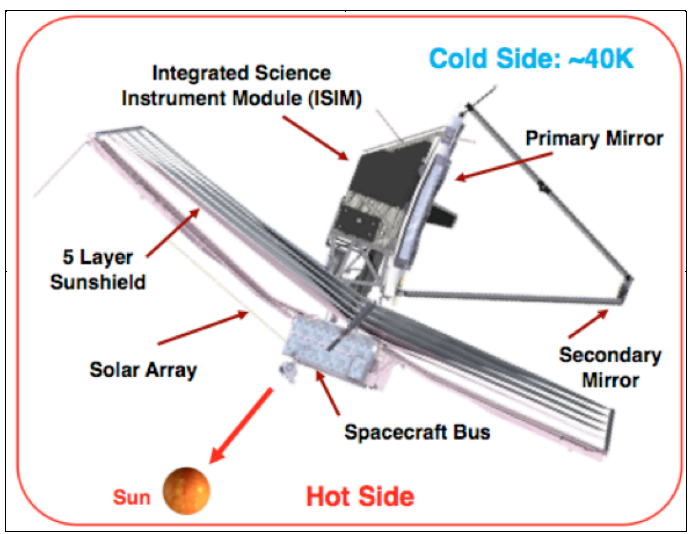

Figure 1: James Webb Space Telescope architecture and passive cooling concept. delivers the required collecting area of $25 \mathrm{~m}^{2}$, the image quality is delivered by the telescope's overall wavefront error, compensation for spacecraft induced jitter, and a stable observatory platform. JWST is groundbreaking in employing a passively cooled architecture to achieve its cryogenic operating temperature, without the need for life-limiting cryogens. The central element of the passive cooling architecture is a five-layer sunshield, where each layer is approximately the size of a tennis court, that ensures the telescope is always in the shade of the sun. Finally, the observatory has to fit into the fairing envelope provided by the Ariane 5, and so the observatory has to be stowed for launch, and subsequently deployed on the way to its final orbit.

Space Telescopes and Instrumentation 2012: Optical, Infrared, and Millimeter Wave, edited by Mark C. Clampin, Giovanni G. Fazio, Howard A. MacEwen, Jacobus M. Oschmann, Jr., Proc. of SPIE Vol. 8442, 84422A

(C) 2012 SPIE · CCC code: 0277-786/12/\$18 - doi: 10.1117/12.926429 


\section{OBSERVATORY DESIGN}

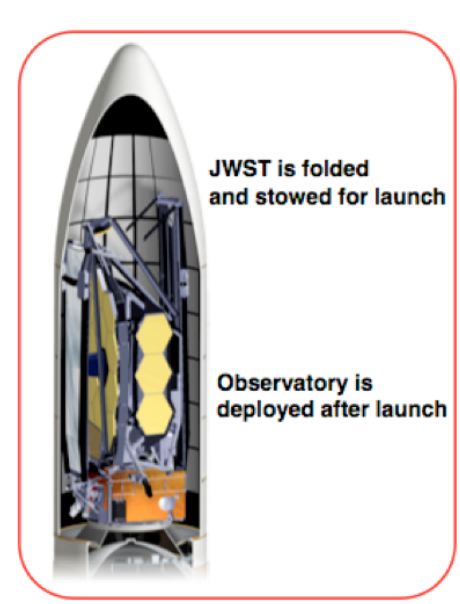

Figure 2: JWST shown in its stowed launch configuration in the fairing of an Ariane 5 launcher.

The James Webb Space Telescope (JWST) is shown schematically in Figure 1. The observatory design breaks down into three zones, the cold side, the sunshield, and the hot side. The observatory cold side comprises the optical telescope element (OTE), and the Integrated Science Instrument Module (ISIM) that houses the science instruments, avionics and radiator panels. The telescope consists of an 18 mirror, segmented primary mirror, a secondary mirror structure, and the aft-optical system (AOS). The primary mirror and secondary mirrors fold and stow for launch, as shown in Figure 2. The telescope's optical sub-systems are mounted to the backbone of JWST, the primary mirror backplane assembly (PMBA). Mounted at the rear of the PMBA is the ISIM that houses the four science instruments and their flight avionics. The cold side hardware interfaces to the spacecraft bus, the primary hot-side hardware element, via a composite tower that is deployed after launch to lift the telescope off the spacecraft bus. The spacecraft bus is built around the observatory's main structural member, a composite cone that houses the propulsion tanks and thrusters. Mounted around the cone are flight avionics, cryocooler, station keeping thrusters, star trackers, radiator shades, inertial reference units, batteries and the communications antennas. The spacecraft bus also serves as the mounting point for a single, deployable solar array. The sunshield comprises five kapton membranes that fold up onto a fore and aft pallet for launch. The sunshield layers are coated with vacuum deposited aluminum, except the layer facing the sun which is coated with a proprietary silicon-based coating. The sunshield pallets fold up and stow around the telescope for launch.

The JWST observatory is launched into orbit around the second Lagrange point L2 via direct insertion. Science operations in this orbit can be conducted 24 hours per day, maximizing the efficiency of scientific observations. The L2 orbit also presents a very benign thermal environment, helping to make JWST a very stable observatory. Operation of JWST at L2 will require routine stationkeeping maneuvers to maintain its orbit, and maneuvers to unload momentum built up as a result of the torque from solar radiation pressure on the sunshield.

\section{OPTICAL TELESCOPE ELEMENT (OTE)}

The major subsystems that form the OTE are broken down and shown schematically in Figure 3. JWST's optical design employs a three mirror anastigmat to deliver the large, well corrected field of view required for deep imaging programs. A fourth mirror, the Fine steering mirror (FSM) is actively controlled and folds the optical chain. The FSM provides image stabilization utilizing guidance signals provided by the Fine Guidance Sensor instrument. The telescope optics are all fabricated from beryllium, which was selected for its light weight, stiffness and thermal stability over the telescope's operating temperature range. The primary mirror is constructed from 18 hexagonal mirror segments, mounted on actuators to provide six degrees of freedom. A seven actuator allows the focus of each segment to be adjusted so that all mirrors are confocal. The secondary mirror is installed on a support structure that is folded and stowed for launch, and locks into place during deployment. The secondary mirror is also mounted on actuators to provide six degrees of freedom. The primary mirror and secondary mirror support structure are both mount to an epoxy-carbon composite structure that holds the mirrors in relative alignment, with each other and with the science instruments. Th structure is known as the primary mirror backplane assembly, and comprises four elements. The backplane is formed from a center section and two hinged wings. The wings fold around the ISIM when stowed for launch, and each support three primary mirror segments. The wings rotate and lock into place during post-launch deployment of the OTE. The backplane mounts 
to the backplane support frame, which also houses the ISIM support structure with the four science instruments.

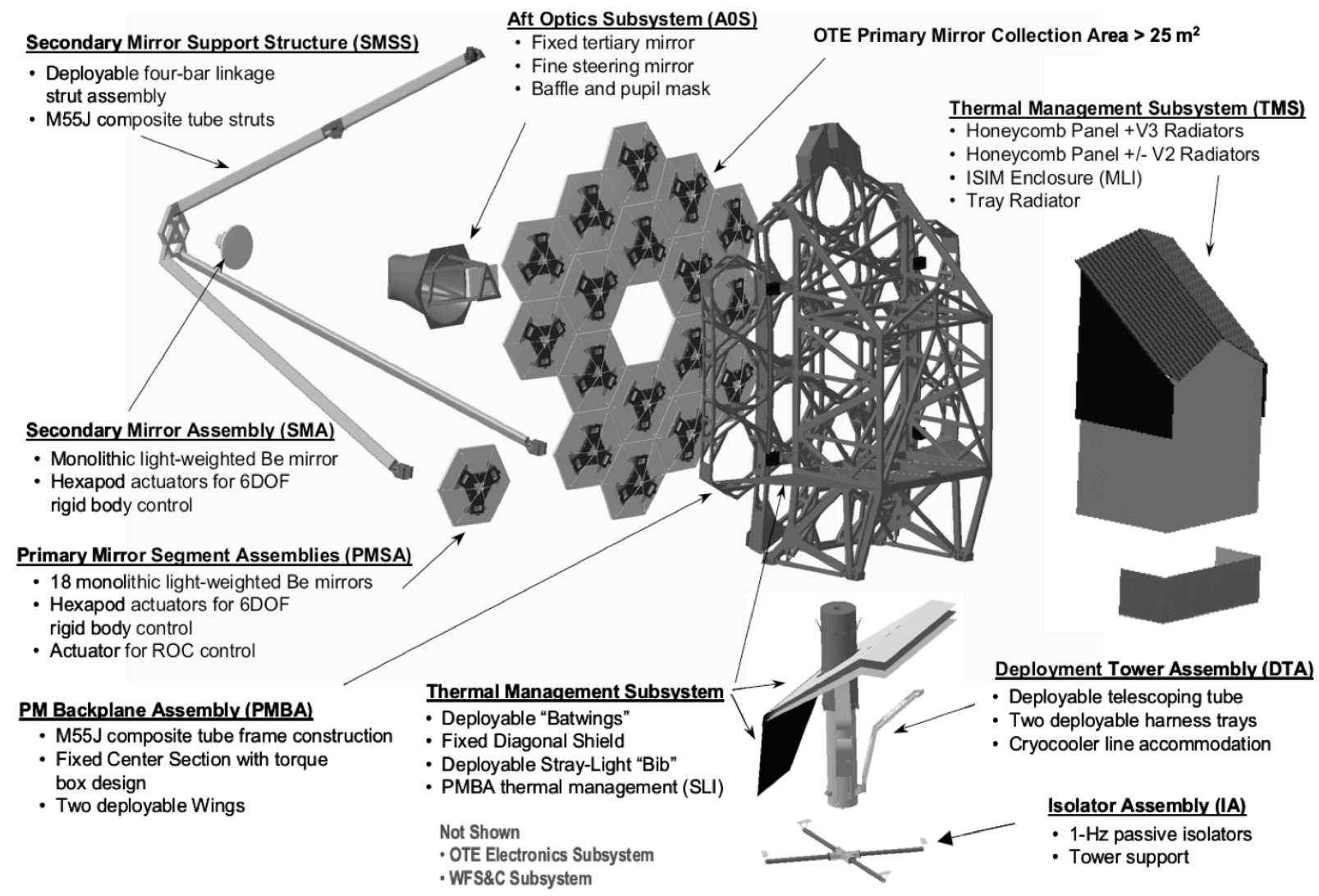

Figure 3: JWST's optical telescope element subsystems

The beryllium mirrors for JWST's telescope have been one of the long lead items for the program and their completion is a significant milestone for the project. Fabrication, polishing and coating of the mirrors was completed in late 2011. Feinberg et al. (2012) describe the steps in the mirror production process. The measured cryogenic surface figures for each of the mirrors are shown in Figure 4, and demonstrate that the mirrors meet their requirements. Simulated images based on results from the mirror's cryogenic acceptance test program show that the optical performance the mirrors are predicted to deliver is diffraction limited imaging at wavelengths $\geq 2 \mu \mathrm{m}$. The image quality at shorter wavelengths, while not diffraction-limited will permit a wide range of science programs, such as stellar populations, to be undertaken at wavelengths down to the mirror's reflectivity cutoff at $\sim 600 \mathrm{~nm}$. JWST's flight mirrors have all received a gold coating to maximize their reflectivity in the infrared (Keski-Kuha et al. 2012). The measured reflectivity for the four mirror optical chain exceeds requirements at every wavelength (e.g. $\sim 93 \%$ at $2.5 \mu \mathrm{m}$ ).

The AOS provides a stable platform for the tertiary and fine steering mirrors, as well as providing stray light baffling. The AOS is built around a beryllium optical bench, and is wrapped in black kapton, as shown in Figure 5. Construction and environmental testing of the AOS is also completed. 


\begin{tabular}{|c|c|c|c|c|}
\hline Mirror & $\begin{array}{c}\text { Measured } \\
\text { (RMS SFE) }\end{array}$ & \multicolumn{1}{c|}{$\begin{array}{c}\text { Uncertainty } \\
\text { (RMS SFE) }\end{array}$} & \multicolumn{1}{c|}{$\begin{array}{c}\text { Total } \\
\text { (RMS SFE) }\end{array}$} & $\begin{array}{c}\text { Requirement } \\
\text { (RMS SFE) }\end{array}$ \\
\hline $\begin{array}{c}\text { 18 primary Segments } \\
\text { (Composite Figure) }\end{array}$ & 23.6 & 8.1 & 25.0 & 25.8 \\
\hline Secondary & 14.7 & 13.2 & 19.8 & 23.5 \\
\hline Tertiary & 18.1 & 9.5 & 20.5 & 23.2 \\
\hline FSM & 13.9 & 4.9 & 14.7 & 18.7 \\
\hline
\end{tabular}
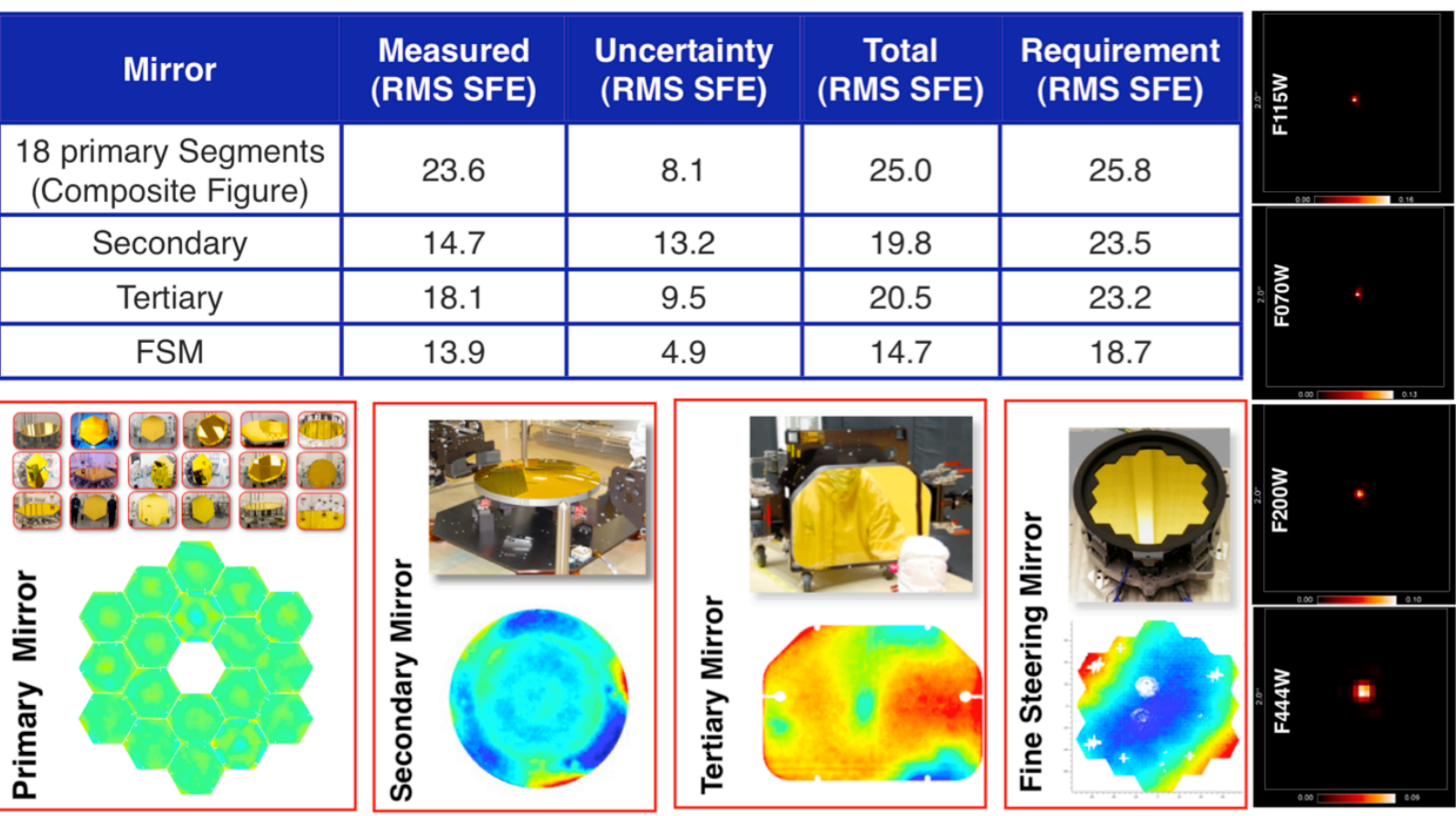

Figure 4: JWSTs flight mirrors have all been completed. Measured surface figure errors are shown above with images of the each mirror's cryogenic surface figure error, and their acceptance test results. On the right we show simulated 2"x2" image boxes, based on initial surface figure measurements from cryogenic testing of the mirror segment A4 (Bowers et al. 2012).

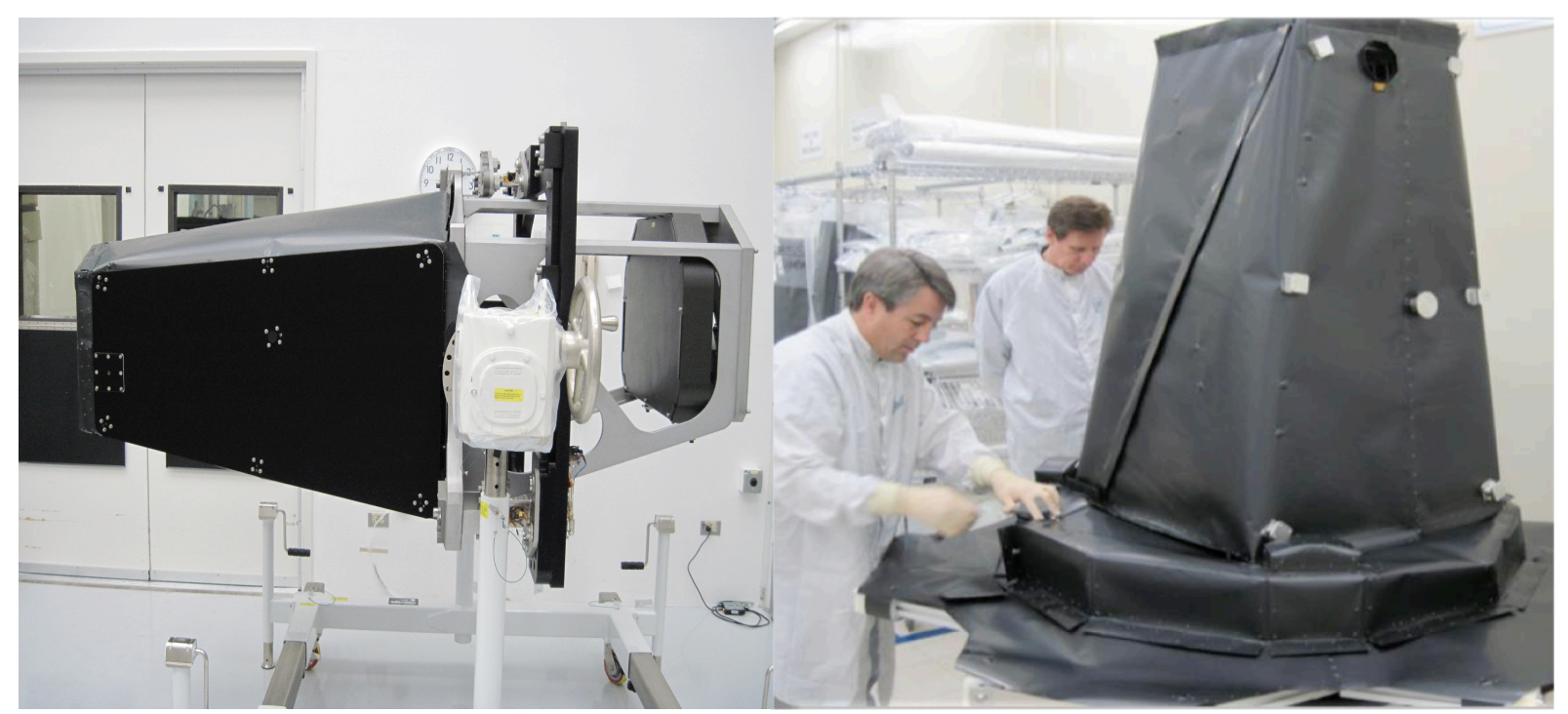

Figure 5: Two views of the aft-optics system as it nears completion at Ball Aerospace. The left view shows the partially wrapped bench with a view of the teriary mirror, while the right hand image shows the full black-kapton wrap around the optical bench.

The primary mirror backplane, the second largest component of the OTE is also making excellent progress towards completion. The center section has been completed, and is awaiting completion of the two wings, and the backplane support frame (BSF). The completed center section is shown at ATK, where it was constructed, in Figure 6. Following completion of these structures, the next major step in the integration and test program will be cryo-cycling at the X-Ray Calibration Facility (XRCF) where the structures will each be tested to the required minimum cryogenic temperature, and optical interface 


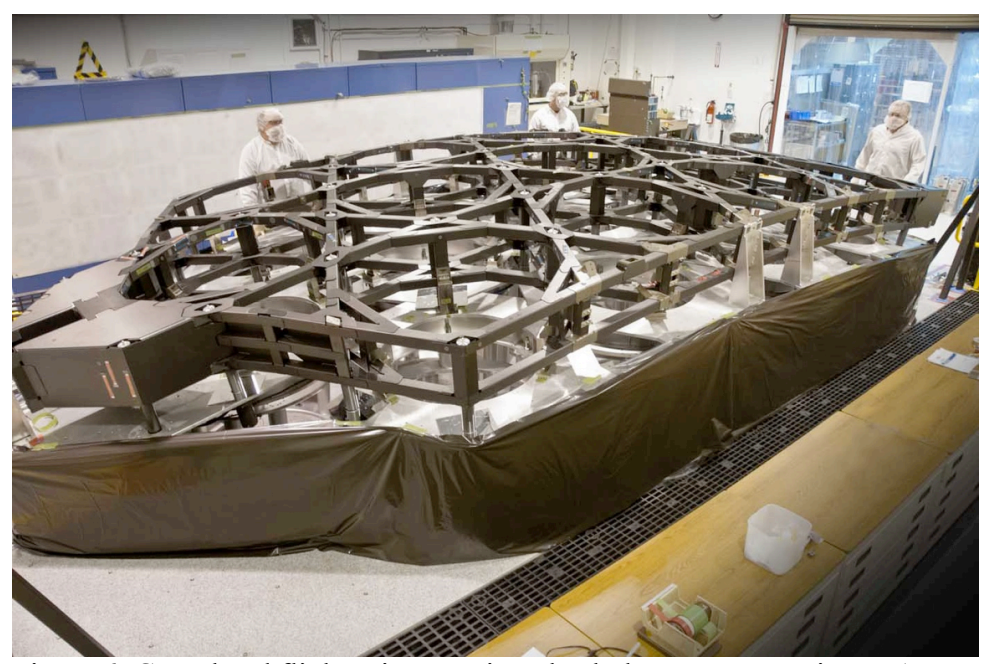

Figure 6: Completed flight primary mirror backplane center section at ATK.

metrology collected at the flight operating temperature. These cryogenic tests will be followed by integration with the flight optics at the Goddard Space Flight Center.

\section{SUNSHIELD}

JWST's sunshield is a significantly different technology from those employed on previous cryogenic missions for space science. The approach adopted by Northrop Grumman (NGAS) in the development of the sunshield has been a series of pathfinder demonstrations which each address an element of the sunshield design implementation. Development is now nearing completion with the production of template membrane layers that are designed to be similar to the final flight membranes in nearly every aspect. The template for membrane layer 3 is shown in Figure 7, fully deployed for testing at Mantech-Nexolve, Huntsville. These template membranes are being used to verify finite element models that predict their shape and alignment tolerances once they are tensioned with their catenary cables. In order to measure the 3-D shape of these tensioned membranes, their surfaces are measured with Lidar. The 3-D shape is important since the membranes have to remain equally spaced apart, and they have steep gradients in the center section, where the sunshield interfaces to the spacecraft bus. The relative alignment of the sunshield edges is also important to verify, since a major mis-alignment between layers could result in the secondary mirror having a view to one of the lower (warmer) membranes in the 5 layer stack, compromising mid-IR sensitivity of the observatory.

In order for JWST to meet its thermal goals the sunshield layers must perform as the thermal models of the observatory predict. To anchor the membrane thermal models, NGAS performed a one 1/3rd scale test

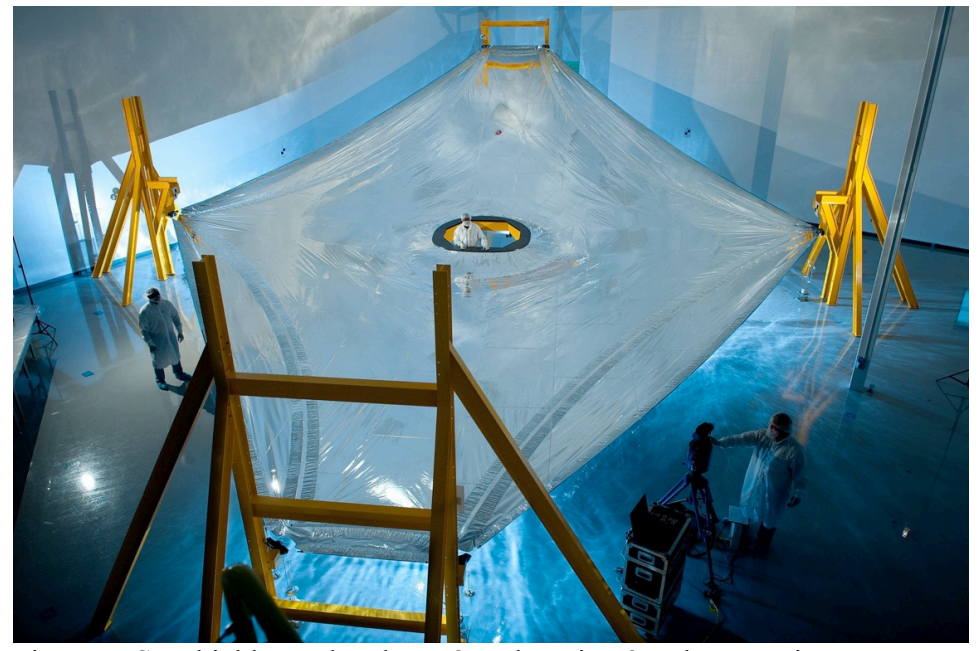

Figure 7: Sunshield template layer 3 undergoing 3D shape testing.

fore/aft sunshield pallets and a mockup of the spacecraft bus. of the 5 membrane layers so that a thermal model could be compared against the realworld performance of the $1 / 3$ rd scale mockup during a thermal balance test. The results of this test which was completed last year, provides confidence that the sunshield will perform as specified, given the excellent correlation of the $1 / 3$ rd scale models and test hardware.

Following shape testing of these membranes, they will be used at NGAS to develop integration and test flows, prior to the arrival of flight hardware. The template membranes will also be used for initial verification of the membrane deployment sequences on a JWST mockup that includes the backplane, the 


\section{SPACECRAFT}

The spacecraft bus is the last hardware element in the development flow for JWST. The spacecraft critical design review is planned for December 2013, and is preceded by design reviews for many of the spacecraft sub-systems. Many of the spacecraft sub-systems, such as the solid state recorder flight unit have already been completed. Other major sub-systems for the spacecraft have engineering design units currently under test.

\section{REFERENCES}

Bowers, Charles W.; Clampin, M.; Feinberg, L.; Keski-Kuha, R.; McKay, A.; Chaney, D.; Gallagher, B.; Ha, K. 2012, American Astronomical Society, AAS Meeting 219, 241.23.

Feinberg Lee D.; Clampin, M.; Keski-Kuha, R.; Atkinson, C.; Texter, S.; Bergeland, M.; \& Gallagher B. B. 2012 this proceedings

Gardner, Jonathan P.; Mather, John C.; Clampin, Mark; Doyon, Rene; Greenhouse, Matthew A.; Hammel, Heidi B.; $\quad$ Hutchings, John B.; Jakobsen, Peter; $\quad$ Lilly, Simon J.; $\quad$ Long, Knox S.; Lunine, Jonathan I.; $\quad$ McCaughrean, Mark J.; $\quad$ Mountain, Matt; $\quad$ Nella, John; $\quad$ Rieke, George H.; Rieke, Marcia J.; $\quad$ Rix, Hans-Walter; $\quad$ Smith, Eric P.; $\quad$ Sonneborn, George; $\quad$ Stiavelli, Massimo; Stockman, H. S.; Windhorst, Rogier A.; Wright, Gillian S. 2006, Space Science Reviews, Volume 123, Issue 4, pp.485-606

Keski-Kuha, R. 2012 this proceedings

\section{ACKNOWLEDGEMENTS}

The JWST system is a collaborative effort involving NASA, ESA, CSA, the Astronomy community and numerous principal investigators. The Observatory benefits from many contributions including NASA GSFC, Northrop Grumman, Ball Aerospace, Alliant Techsystems, ITT Exelis, and Nexolve. 